ISSN: $1130-2887$

DOI: http://dx.doi.org/10.14201/alh2014666994

\title{
IDEOLOGÍA PARTIDARIA, COMPETENCIA ELECTORAL Y ELECCIÓN DE LEGISLADORAS EN CINCO DEMOCRACIAS LATINOAMERICANAS: ARGENTINA, BRASIL, CHILE, PERÚ Y URUGUAY, 1980-2013 Party Ideology, Electoral Competition and the Election of Women in Five Latin American Democracies: Argentina, Brasil, Chile, Peru and Uruguay, 1980-2013
}

\author{
Santiago ALLES \\ Rice University, Estados Unidos \\ $\triangle$ santiago.alles@rice.edu
}

BIBLID [1130-2887 (2014) 66, 69-94]

Fecha de recepción: 18 de marzo del 2013

Fecha de aceptación y versión final: 12 de enero del 2014

RESUMEN: Las mujeres han hecho enormes progresos en su incorporación a la arena política. No obstante, el ritmo de esa incorporación ha sido desigual. El presente artículo analiza los efectos de la ideología partidaria y de las características de la competencia electoral sobre la elección de mujeres en cargos legislativos, en cinco democracias latinoamericanas a lo largo de tres décadas. Los resultados brindan evidencia del efecto de factores partidarios (ideología) e institucionales (tipos de lista), al tiempo que pone en duda el efecto de otros factores aseverados por la literatura, tales como la magnitud de distrito y la magnitud partidaria.

Palabras clave: elección de mujeres, ideología partidaria, magnitud partidaria, tipo de lista.

ABSTRACT: Women have made enormous progress in their political incorporation. However, the pace of that incorporation has differed across countries. The present article analyzes the effects of party ideology and of the characteristics of electoral competition on the election of women in legislative seats in five Latin American democracies over three decades. The empirical results provide evidence of the effects of party (ideology) and institutional factors (type of ballot); 
and at the same time, call into question the effect of factors previously asserted in the literature, such as district magnitude y party magnitude.

Key words: women election, party ideology, party magnitude, type of ballot.

\section{INTRODUCCIÓN ${ }^{1}$}

Las mujeres han hecho enormes progresos en materia de derechos y de incorporación a la arena política desde inicios del siglo XX. Sin embargo, recién en décadas recientes, esos derechos se han convertido en posiciones de poder: las mujeres ocupan hoy una de cada cinco bancas legislativas en las Américas (Inter-Parliamentary UnionIPU-2013). Esa mayor presencia legislativa está asociada con legislación más favorable a los intereses de las mujeres (Childs y Krook 2009; Schwindt-Bayer 2006; Franceschet y Piscopo 2008; Jones 1997; Kittilson 2008), como así también con la emergencia de modelos a imitar (Wolbrecht y Campbell 2007). ¿Cómo se han logrado tales progresos? La introducción de cuotas de género ha sido considerado por la literatura como una «vía rápida» (Dahlerup y Freidenvall 2005; Tripp y Kang 2008) a la igualdad entre hombres y mujeres. A su vez, en la literatura existe también consenso acerca de los efectos positivos de las reglas proporcionales sobre las oportunidades de las mujeres en comparación con las reglas mayoritarias (Paxton et al. 2007).

No obstante, más allá de tales acuerdos sobre cuotas y reglas proporcionales, los efectos de otros factores explicativos son aún poco claros. En primer lugar, ¿cuál es el efecto de la ideología partidaria sobre la elección de mujeres? Los partidos de izquierda, con un programa ideológico en el cual la igualdad ocupa un espacio sustantivo, deberían ser más favorables a la selección de mujeres en sus listas de candidatos; al tiempo que sus votantes también deberían ser más receptivos a esa oferta. En ese sentido, la mayor inclusión de mujeres en la política reciente ¿ha sido resultado del giro a la izquierda en la política latinoamericana (Levitsky y Roberts 2011; Weyland et al. 2010; Remmer 2012) y, en consecuencia, de miradas más igualitarias de la política? En esa misma dirección, las presidencias de mujeres socialistas como Michelle Bachelet o Dilma Rousseff encajan muy bien en este argumento. Sin embargo, la respuesta no es obvia. Por un lado, la introducción de las cuotas de género en la región ocurrió durante el ascenso y esplendor de la era neoliberal. Por otro lado, el espacio que las mujeres

1. El autor agradece a Mark P. Jones, Randy Stevenson, Tiffany Barnes, Carolina Tchintian, Justin Esarey, Julia Rubio y Matt Loftis, como así también a los participantes de respectivos paneles en Southwestern Social Science Association Conference (New Orleans, Louisiana: 27-30 de marzo, 2013) y en American/Comparative Workshop (Department of Political Science, Rice University) por sus valiosos comentarios y sugerencias a versiones previas del presente artículo. Agradece también por el apoyo al Instituto de Ciencias Políticas y Relaciones Internacionales (UCA, Argentina), en especial a Enrique Aguilar y Marcelo Camusso, donde fue realizada parte de la investigación. Por último, agradece a dos evaluadores anónimos de América Latina Hoy, Revista de Ciencias Sociales por sus contribuciones. La base de datos para replicar el presente trabajo, junto al libro de códigos y las fuentes, se encuentran disponibles en: <http://santiago-alles.net/research $>$. 
ocupan, para continuar el ejemplo, en el Partido Socialista (PS) chileno o el Partido de los Trabajadores (PT) brasileño es menor comparado con otros partidos de centro como el peronismo argentino.

Por otra parte, además de la ideología partidaria, otros dos interrogantes no tienen respuestas claras en la literatura. ¿Cuál es el efecto de la magnitud del distrito sobre las chances de las mujeres? La literatura ha indicado que los distritos electorales grandes presentan menores barreras a la elección de mujeres. No obstante, la evidencia no es uniforme: trabajos más recientes no han encontrado ningún efecto sustantivo. ¿Cuál es el efecto del tipo de lista sobre la elección de mujeres? Una parte de la literatura argumenta que las listas abiertas permiten a los votantes reordenar la oferta partidaria y así las mujeres no quedan atrapadas en el orden de candidaturas decidido por las autoridades partidarias. Sin embargo, otra parte de la literatura responde indicando que tal posibilidad de establecer lazos más estrechos con los votantes es minimizada por los costos de la mayor competencia intrapartidaria en las elecciones con lista abierta.

El presente artículo tiene algunas fortalezas sobre trabajos precedentes. La literatura comparada sobre elección de mujeres ha sido criticada por cierta inconsistencia entre teoría y evidencia, donde datos agregados se usan para evaluar explicaciones teóricas diseñadas a nivel del distrito (Archenti y Tula 2008) y por observar un único instante en el tiempo, omitiendo la naturaleza histórica del proceso de incorporación (Hughes y Paxton 2007; Roberts et al. 2013). Por el contrario, el presente trabajo descansa sobre datos a nivel del distrito, incluyendo todos los legisladores (diputados y senadores) electos desde la primera elección legislativa postransición: 1980 en Perú, 1983 en Argentina, 1984 en Uruguay, 1986 en Brasil y 1989 en Chile. Así, se realiza el análisis gracias a un set de datos que abarca la elección de 9.340 legisladores en un variado espectro de diseños institucionales, condiciones socioeconómicas e ideologías partidarias a lo largo de tres décadas. Con estos datos, este artículo contribuye a resolver esos tres debates irresueltos en la literatura comparada.

El resto del artículo está organizado de la siguiente manera. Primero se presenta una breve revisión de la literatura sobre elección de mujeres, a fin de enmarcar las preguntas de investigación en el debate teórico. Luego, se presenta el diseño de investigación y las decisiones operativas adoptadas en la definición de los datos y, a continuación, se presenta el modelo estadístico utilizado y se reportan los resultados más significativos. En la sección final se discuten algunas implicancias teóricas.

\section{LEGISLADORAS, IDEOLOGÍA E INSTITUCIONES: UNA REVISIÓN DEL DEBATE}

Las reglas proporcionales y las cuotas de género han sido señaladas consistentemente como factores favorables a la elección de mujeres en cargos legislativos. Por un lado, la literatura ha indicado, siguiendo el argumento seminal propuesto por Duverger, que las reglas electorales mayoritarias reducen las probabilidades de elección de mujeres, mientras que la representación proporcional ofrece un escenario más favorable (Matland 1998; Kenworthy y Malami 1999; Norris 2004; Norris 1985; Reynolds 1999; Paxton 1997; Paxton y Kunovich 2003). Las ventajas de las reglas proporcionales 
EN CINCO DEMOCRACIAS LATINOAMERICANAS...
son, tal como han argumentado Paxton y coautores (2007: 269, la traducción es propia), quizás el hallazgo más consistente y mejor documentado en el análisis comparado sobre mujeres en política, aun cuando tampoco se encuentre libre de críticas (Roberts et al. 2013).

Las cuotas de género han sido señaladas como un mecanismo crucial para incrementar el número de mujeres electas en órganos legislativos (Htun y Jones 2002; Jones 2009, 1998, 2004; Htun 2002; Dahlerup 1988, 2003; Dahlerup y Freidenvall 2005; Jones et al. 2012; Tripp y Kang 2008; Norris 2004; Alles 2008, 2007). No obstante, su diseño juega un papel clave en su eficacia: tanto los mandatos de posición (Jones 2004; Schwindt-Bayer 2010) como su implementación por medio de sanciones (Dahlerup 2005) tienen una influencia decisiva en la efectividad de las cuotas. Más aún, algunos trabajos han indicado que las cuotas tienen efectos de largo plazo: los efectos iniciales aumentan por su implementación sostenida (Matland 1993; Matland y Studlar 1998; Alles 2007) y aun cuando las cuotas son eliminadas, las mujeres gozan de mejores oportunidades (Bhavnani 2009).

Más allá del diseño de las instituciones, los partidos políticos ocupan un papel principal en el proceso electoral: los partidos tienen control sobre el proceso de selección de candidatos y sobre los recursos necesarios para llevar adelante una campaña electoral (Norris y Lovenduski 1995). De esa manera, ocupan un lugar principal en la incorporación de nuevos actores en la arena electoral. Las características de los partidos políticos pueden afectar la elección de mujeres a través de tres mecanismos causales: (a) sus élites partidarias, (b) sus reglas de nominación y (c) su ideología. Primero, la composición de las élites partidarias puede influir sobre la nominación de los candidatos partidarios y la distribución de los recursos de campaña: líderes mujeres pueden ser más favorables a la nominación de mujeres (Kunovich y Paxton 2005).

Segundo, cuando las reglas de nominación son más centralizadas (Hazan y Rahat 2010, 2001; Freidenberg y Alcántara Sáez 2009), la dirigencia partidaria está mejor dotada para responder a las demandas de los electores y así incluir un mayor número de mujeres cuando los electores son favorables a la igualdad de género (Caul 1999). Tercero, los partidos de izquierda son proclives a miradas igualitarias en temas de género, minorías étnicas y otros grupos subrepresentados, por lo cual estos partidos estarán más abiertos a incluir mujeres como activistas y en puestos de liderazgo (Caul 1999); y serán más favorables a la inclusión de grupos históricamente excluidos en las listas de candidatos (Matland y Studlar 1996). Los partidos de izquierda pueden, entonces, operar como canales de inclusión de las mujeres. De esto se desprende la siguiente hipótesis de trabajo:

H1. Las probabilidades de elección de mujeres aumentan a medida que las posiciones ideológicas de los partidos están más a la izquierda.

La literatura ha indicado con insistencia que los distritos electorales grandes presentan menores barreras a la elección de mujeres (Matland 1993; Matland y Taylor 1997; Norris 2004; Jones 1998; Rule 1987; Paxton et al. 2007). Los sistemas de 

EN CINCO DEMOCRACIAS LATINOAMERICANAS. .

representación proporcional son considerados superiores en su apertura a la inclusión de mujeres por (aunque no exclusivamente) el uso de distritos plurinominales. No obstante, la evidencia ha comenzado a poner en duda el efecto positivo de la magnitud: los efectos de la magnitud serían, según trabajos más recientes (Jones 2009; Schmidt 2009; Jones et al. 2012), condicionales al tipo de lista empleada y al uso de sistemas de cuotas adecuadamente diseñados.

El tamaño de los distritos en sistemas proporcionales está vinculado con la elección de mujeres a través de mecanismos causales indirectos. Por un lado, los distritos grandes están asociados con delegaciones partidarias más grandes, lo cual reduce los juegos de suma cero intrapartidarios y facilita así la selección de un grupo de candidatos más diverso (Matland 2002). Más aún, en elecciones mayoritarias, estos juegos de suma cero son difíciles de romper aun cuando las mujeres acceden a posiciones de poder en el partido: las chances de una mujer de resultar nominada en competencias mayoritarias no crecen en presencia de mujeres en la cúpula partidaria, como sí ocurre en elecciones proporcionales (Kunovich y Paxton 2005).

Por otro lado, la magnitud de distritos está asociada con mayores niveles de fragmentación partidaria (Cox 1997; Taagepera y Shugart 1989); y la mayor diversidad en la oferta electoral facilita la presión de las mujeres en reclamo de espacios políticos y, en definitiva, el «contagio» (Matland y Studlar 1996) entre partidos: la exclusión de las mujeres es más evidente cuando ya han sido incluidas por algún partido «pionero», de manera que los partidos más resistentes a su inclusión se exponen a castigos electorales. En definitiva, si la magnitud de los distritos tiene efecto sobre la elección de mujeres, ese efecto tiene que poder desmontarse en sus componentes causales. Esto supone la siguiente hipótesis de trabajo:

H2. Las probabilidades de elección de mujeres están asociadas con (a) mayores magnitudes partidarias mayores y (b) mayor fragmentación electoral.

El tipo de boleta puede afectar las oportunidades electorales de las mujeres por tres mecanismos causales. Primero, las listas abiertas incrementan la competencia intrapartidaria, lo cual otorga ventaja a los competidores con más recursos y a los titulares en busca de reelección (Carey y Shugart 1995; Ames 1995), y deja a las mujeres en desventaja: la evidencia ha señalado que las mujeres, tal como ocurre con otros grupos de recién llegados a la arena política, disponen de menores recursos de campaña (Marx et al. 2007; Htun y Jones 2002). En elecciones con listas cerradas, contrariamente, no hay competencia intrapartidaria y las mujeres no pueden verse perjudicadas por las decisiones sobre asignación de recursos partidarios. Segundo, en elecciones con listas abiertas en arenas donde predomina una cultura tradicional, las autoridades partidarias nominan menos mujeres porque el género no transmite un valor distintivo frente a los votantes y, por el contrario, puede dañar las chances del partido (Valdini 2012).

Finalmente, la efectividad de las cuotas puede ser condicional al tipo de listas utilizadas en la elección: tanto la implementación de mandatos de posición como 
EN CINCO DEMOCRACIAS LATINOAMERICANAS...
de sanciones son más difíciles (y en algunos casos imposibles) en elecciones con listas abiertas. No obstante, a pesar de estos argumentos, los efectos del tipo de lista son aun motivo de controversias en la literatura: numerosos trabajos (Schmidt 2009; Matland 2006 y 2005; Schmidt y Saunders 2004; Shugart 1994) han criticado la aparente superioridad de las listas de candidatos cerradas; mientras otros (Jones 2009; Htun y Jones 2002; Jones et al. 2012; Schwindt-Bayer 2010; Norris 2004) han presentado nueva evidencia sobre los efectos negativos de las listas abiertas sobre la elección de mujeres. Esto supone una nueva hipótesis:

H3. Las listas abiertas están asociadas con menores probabilidades de elección de mujeres.

En la próxima sección se presenta el diseño de investigación y, a continuación, en el siguiente apartado, el modelo estadístico empleado para analizar los datos.

\section{DISEÑO DE INVESTIGACIÓN: SELECCIÓN DE CASOS, VARIABLES Y DATOS}

\section{III.1. Selección de casos}

La literatura comparada sobre elección de mujeres ha seguido con frecuencia un mismo modelo en términos de diseño de investigación: (a) un extenso número de casos, (b) observados en un único momento en el tiempo, (c) utilizando la proporción de mujeres en el Congreso/Parlamento como variable dependiente y (d) promedios nacionales de los factores de interés como variables independientes, (e) analizados por medio de modelos lineales (Kenworthy y Malami 1999; Reynolds 1999; Norris 2004; Paxton y Kunovich 2003; Paxton 1997; Kunovich y Paxton 2005; Schmidt 2009; Matland 1998; Rule 1987; Inglehart et al. 2002). Las variaciones hechas sobre este modelo, con frecuencia, han extendido el rango temporal pero han conservado el uso de datos agregados (Schwindt-Bayer 2009; Roberts et al. 2013; Fallon et al. 2012; Salmond 2006; Rosenbluth et al. 2006).

La definición de la muestra parece regir esta literatura, mientras la definición operativa de las variables ha sido resultado de aquella selección de casos: dadas las dificultades para la recolección de datos en trabajos con $\mathrm{N}$ grande, el uso de datos agregados al nivel nacional es una solución razonable. Sin embargo, estas decisiones en el diseño tienen implicancias considerables.

La incorporación de mujeres ha seguido diferentes patrones históricos en diferentes casos (Paxton y Hughes 2007). Los diseños basados en una única observación pueden implicar, tal como señalan Roberts y coautores (2013), «mirar en el momento equivocado» (la traducción es propia) porque el timing de la inclusión difiere de un país a otro: a pesar de las demandas en favor de diseños longitudinales (Hughes y Paxton 2007), su uso ha sido sólo reciente (Roberts et al. 2013; Fallon et al. 2012; Paxton et al. 2010; Paxton et al. 2006). Además, el uso de información agregada implica cierta inconsistencia 

EN CINCO DEMOCRACIAS LATINOAMERICANAS. .

entre teoría y evidencia: mientras las explicaciones teóricas han sido diseñadas al nivel del distrito electoral, los test con $\mathrm{N}$ grande emplean información agregada a nivel nacional. Mientras esta literatura, en pos de incrementar la cantidad de observaciones (King et al. 1994), acepta cierto sacrificio en la calidad de los datos, tanto los estudios de caso (Jones 2004; Schmidt y Saunders 2004; Matland 1993; Matland y Studlar 1996 y 1998; Matland y Taylor 1997; Jones y Navia 1999; Alles 2008, 2007; Welch y Studlar 1990) como los estudios de área con N medianas (Jones 2009; Jones et al. 2012) permiten una mejor correspondencia entre teoría y datos utilizando distritos, listas partidarias o legisladores individuales como unidades de análisis. En este sentido, el presente artículo recoge tanto las preocupaciones por el timing (Roberts et al. 2013) como las ecológicas (Archenti y Tula 2008); razón por la cual el diseño de investigación descansa sobre una base de datos de 9.340 legisladores (véase Tabla I), electos en 4.191 listas partidarias, elaborada al nivel del distrito, en un periodo de tres décadas.

TABLA I

NÚMERO DE LEGISLADORES ELECTOS SEGÚN PAÍS Y SEGÚN CÁMARA (1980-2013)`

\begin{tabular}{lccc}
\hline & DIPUTADOS & SENADO & TOTAL \\
\hline Argentina & 2.177 & 216 & 2.393 \\
Brasil & 3.555 & 296 & 3.851 \\
Chile & 840 & 152 & 992 \\
Perú & 1.150 & 180 & 1.330 \\
Uruguay & 594 & 180 & 774 \\
\hline Total & 8.316 & 1.024 & 9.340 \\
\hline
\end{tabular}

Fuente: Elaboración propia.

Los diseños de investigación con un número limitado de casos, tal como ha sido señalado en trabajos seminales sobre análisis comparado (Lijphart 1971), exigen un uso cuidadoso de la similitud y la diferencia. La selección de casos en el presente artículo ha sido diseñada para (a) cubrir un variado espectro de diseños institucionales y (b) mantener constantes factores culturales. La diversidad de los casos seleccionados, por un lado, introduce una considerable variación de elementos institucionales teóricamente sustantivos: los legisladores en la muestra han sido elegidos con y sin cuotas de género; por medio de listas abiertas y cerradas ${ }^{2}$, tanto en distritos pequeños, medianos y grandes.

2. Los legisladores en Uruguay se eligen por medio de listas por facción, conocidas usualmente como «Lemas» o, más técnicamente, como DVS (D. BUQUET 2003; M. JONES 2000). Este tipo de listas son con frecuencia clasificadas como listas cerradas en la literatura sobre sistemas electorales (G. COX 1997). Más importante aún, esta definición operativa coincide con la brindada por los trabajos más 
SANTIAGO ALLES EN CINCO DEMOCRACIAS LATINOAMERICANAS...

TABLA II

REGLAS ELECTORALES PARA LA ELECCIÓN DE LEGISLADORES NACIONALES (1980-2013)

\begin{tabular}{|c|c|c|c|}
\hline \multirow{2}{*}{$\begin{array}{l}\text { MAGNITUD } \\
\text { DE DISTRITO }\end{array}$} & \multirow{2}{*}{$\begin{array}{c}\text { TIPO } \\
\text { DE LISTA }\end{array}$} & \multicolumn{2}{|c|}{ CUOTAS DE GÉNERO } \\
\hline & & No & Si \\
\hline \multirow{2}{*}{ 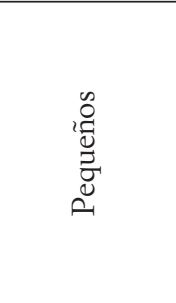 } & Abierta & $\begin{array}{l}\text { Brasil-S }(1986,1994) \\
\text { Chile-D } \\
\text { Chile-S } \\
\text { Perú-D (1985-1990) }\end{array}$ & $\begin{array}{l}\text { Brasil-S }(2002,2010) \\
\text { Perú-D (2001-2011) }\end{array}$ \\
\hline & Cerrada & $\begin{array}{l}\text { Argentina-D (1983-1991) } \\
\text { Brasil-S (1990) } \\
\text { Perú-D (1980-1990) } \\
\text { Uruguay-D }\end{array}$ & $\begin{array}{l}\text { Argentina-S (2001-2013) } \\
\text { Argentina-D (1993-2013) } \\
\text { Brasil-S (1998, 2006) }\end{array}$ \\
\hline \multirow{2}{*}{$\overbrace{\substack{0 \\
\infty}}^{\infty}$} & Abierta & $\begin{array}{l}\text { Brasil-D (1986-1994) } \\
\text { Perú-D (1985-1990) }\end{array}$ & $\begin{array}{l}\text { Brasil-D (1998-2010) } \\
\text { Perú-D (2001-2011) }\end{array}$ \\
\hline & Cerrada & $\begin{array}{l}\text { Argentina-D (1983-1991) } \\
\text { Perú-D (1980) } \\
\text { Uruguay-D }\end{array}$ & Argentina-D (1993-2013) \\
\hline \multirow{2}{*}{ 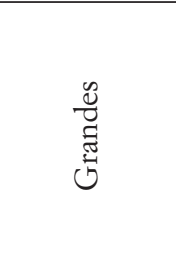 } & Abierta & $\begin{array}{l}\text { Brasil-D (1986-1994) } \\
\text { Perú-D (1985-1990) } \\
\text { Perú-S (1985-1990) }\end{array}$ & $\begin{array}{l}\text { Brasil-D (1998-2010) } \\
\text { Perú-D (2000-2011) }\end{array}$ \\
\hline & Cerrada & $\begin{array}{l}\text { Argentina-D (1983-1991) } \\
\text { Perú-D (1980) } \\
\text { Perú-S (1980) } \\
\text { Uruguay-S }\end{array}$ & Argentina-D (1993-2013) \\
\hline
\end{tabular}

Nota: Las elecciones de senadores en Brasil en que se eligió un solo escaño y las elecciones de diputados en Perú en que se eligió un solo escaño y los partidos no excedieron ese número de candidatos (1985-1990), como así también las elecciones en Uruguay mediante listas por facción fueron consideradas como listas cerradas.

Los senadores en Argentina han sido elegidos directamente desde 2001. Bajo la Constitución previa, los senadores eran designados por las legislaturas provinciales, por lo cual fueron excluidos del análisis.

Fuente: Elaboración propia.

Tal variedad institucional en la selección de casos, resumida en la Tabla II, es crucial para controlar la mayor parte de las explicaciones propuestas para la elección de mujeres, incluyendo cuotas de género, magnitud de distrito y el tipo de lista. Más aún, las legislaturas son bicamerales en todos los casos seleccionados (excepto Perú entre 1995

recientes participantes en el debate (M. JONES 2009; G. SCHMIDT 2009): los votantes no pueden alterar el orden de candidatos ofrecido, lo cual constituye el mecanismo causal en el argumento teórico que conecta tipo de lista y oportunidades de las mujeres. En suma, en el análisis empírico se las considerará como una lista cerrada. 

EN CINCO DEMOCRACIAS LATINOAMERICANAS. .

y 2011), por lo cual en el presente análisis se incluyen tanto diputados como senadores, lo cual es inusual en una literatura largamente basada en elecciones de diputados. Por otro lado, el foco exclusivo en cinco casos latinoamericanos permite controlar factores culturales que han sido considerados importantes (Inglehart y Norris 2003; Paxton y Kunovich 2003; Matland 2006) tanto para explicar el nivel de representación de mujeres como el funcionamiento de las cuotas de género.

\section{III.2. Modelo multivariado}

El objetivo central del presente artículo es brindar una explicación de la elección de mujeres en bancas legislativas. Un modelo de regresión binomial reproduce el proceso estocástico de generación de datos: el resultado es el producto de una serie de experimentos Bernoulli, es decir, una serie de loterías que pueden adoptar uno de dos posibles resultados entre sí mutuamente excluyentes. Por una parte, el número de mujeres electas es resultado de $k$ opciones binarias sobre $n$ delegaciones partidarias en un distrito electoral dado. Por otra parte, gracias a su componente logístico, el modelo permite la incorporación en el análisis de variables en diferentes niveles (partidario, distrital y nacional) y, desde esos factores independientes, estimar la probabilidad de elegir una mujer en diferentes contextos.

Sin embargo, este tipo de modelo descansa en algunos supuestos: un modelo logístico asume que cada legislador electo es una observación independiente, lo cual implica que el resultado de cada elección es independiente (a) de la elección en otros países; (b) de los resultados en otros distritos del mismo país; y también (c) de los resultados anteriores y posteriores en el mismo distrito. El último supuesto puede resultar demasiado exigente para algunos observadores porque el porcentaje de mujeres electas puede estar asociado con factores idiosincráticos locales no observados. Sin embargo, este supuesto sólo implica que los votantes son libres de tomar una nueva decisión en cada oportunidad; mientras que el efecto potencial de factores idiosincráticos puede ser capturado a través de efectos fijos en el modelo. Una vez que estos supuestos son admitidos, los datos de panel pueden ser tratados como puramente comparados (Train 2009; Gelman y Hill 2007).

El término ij indica una delegación partidaria en una elección legislativa $i$, en un país $j$. Entonces, el componente estocástico del análisis estadístico puede ser capturado por una distribución de probabilidad tal como:

$$
\mathcal{L}\left(y_{i j} \mid \varphi_{i j}, n\right)=\left(\begin{array}{c}
n \\
y_{i j}
\end{array}\right) \varphi_{i j}^{y_{i j}}\left(1-\varphi_{i j}\right)^{n-y_{i j}}
$$

donde el término $y_{i j}$ es un valor discreto que indica el número de mujeres electas en $k$ escaños obtenidos por el partido $n$ en la elección ij. Así, el componente sistemático del modelo es un término logístico, tal como: 


$$
\varphi_{i j}=\frac{1}{1+e^{-g(\beta, X)}}
$$

donde $g(\cdot)$ es una función lineal integradora, que introduce las variables explicativas en el modelo estadístico:

$$
g(\beta, X)=\alpha+\beta X_{i j}+\gamma_{j}+\varepsilon_{i j}
$$

El término $\beta$ representa los coeficientes que capturan el efecto del vector $X$ de variables independientes sobre $y_{i j}$ en la observación $i j$, mientras $\alpha$ es una constante, $\gamma_{j}$ es un término de efecto fijo que captura las características idiosincráticas en el país $j$; y $\varepsilon_{i j}$ es un término de error para la observación $i j$.

Los modelos que no controlan efectos fijos, por el contrario, se simplifican al no incluir el término $\gamma_{j}$ y reducir la función integradora a:

$$
\alpha+\beta X_{i}+\varepsilon_{i}
$$

Esto tiene la ventaja de minimizar potenciales problemas de multicolinealidad entre variables medidas a escala nacional y factores idiosincráticos, pero al mismo tiempo deja esos factores idiosincráticos como elementos no controlados.

\section{III.3. Variables y datos}

El vector $X$ de variables independientes incluye factores en diferentes niveles: (a) variables partidarias; (b) variables distritales; y (c) variables nacionales.

\section{III.3.1. Variables partidarias}

Ideología de las bases partidarias. Colomer y Escatel (2005) han medido la posición ideológica de los partidos latinoamericanos entre 1995 y 2002 a partir de la identificación ideológica de sus votantes. La posición ideológica ocupada por cada partido no varía entre elecciones, sino que por el contrario se asume constante 3 . El indicador está basado en encuestas de Latinobarómetro y la escala del indicador varía de 0 a 10,

3. El uso de esta medida de ideología partidaria descansa en dos supuestos. Por un lado, el indicador asume que las posiciones partidarias son estables: los partidos representan el mismo espacio ideológico a lo largo del periodo observado. Por otro lado, la medida asume que los votantes (entrevistados) en diferentes países comparten la misma escala ideológica. Aun cuando este supuesto puede ser poco realista en un trabajo comparado, evidencia reciente (E. ZECHMEISTER y M. CORRAL 2013) indica que a lo largo de la región existe cierto significado compartido de los términos izquierda-derecha, aunque sujeto a factores contextuales específicos. 

EN CINCO DEMOCRACIAS LATINOAMERICANAS. .

donde 10 es la posición extrema derecha. El indicador provee información para 6.785 legisladores $(72,6 \%$ de la población analizada).

Magnitud partidaria. La magnitud de partido es el número de escaños ganados por un partido en un distrito dado.

\section{III.3.2. Variables distritales}

Fragmentación electoral. El número efectivo de partidos (Laakso y Taagepera 1979) ha sido estimado según la distribución de (a) votos y (b) escaños para cada distrito electoral, en la elección correspondiente.

Analfabetismo. La tasa de analfabetismo es el porcentaje de personas (de 10 años y más) que no saben leer y escribir, sobre el total de habitantes en el grupo de edad, en el distrito electoral, a partir de la información censal más reciente. La tasa de analfabetismo se utiliza como variable de control de las diferencias socioeconómicas entre distritos electorales: a mayores tasas de analfabetismo, menores niveles de desarrollo social en el distrito. La tasa de analfabetismo está disponible para todos los distritos electorales.

\section{III.3.3. Variables nacionales}

Tipo de lista. Los legisladores han sido elegidos usando diferentes tipos de listas de candidatos: el tipo de lista es capturado en el modelo por medio de variables dicotómicas.

Cámara legislativa. La selección de casos incluye 8.316 diputados y 1.024 senadores. La cámara legislativa es capturada en el modelo a través de una variable dicotómica.

Cuotas de género. El uso de cuotas es capturado en el modelo por medio de una variable dicotómica: aproximadamente la mitad de los legisladores (4.332 legisladores, $46,4 \%$ de las observaciones) fueron elegidos bajo leyes de cuotas.

Nivel de democracia. El índice de Freedom House provee un indicador del nivel de democracia comparable tanto entre países como a lo largo del periodo. El valor de la variable es un promedio de dos puntajes, «libertades civiles» y «derechos políticos»; y la escala varía de 1 a 7 , donde 7 es el extremo autoritario.

\section{EVIDENCIA: DIPUTADAS Y SENADORAS EN CINCO DEMOCRACIAS}

El reconocimiento de los derechos políticos de las mujeres ha sido el resultado de una larga lucha del movimiento feminista (Molyneux 2001). Los derechos electorales de las mujeres fueron reconocidos a lo largo de las décadas de 1930 y 1940 en la mayor parte de América Latina (Schwindt-Bayer 2010: 43), no obstante lo cual el acceso de mujeres a posiciones de poder no fue inmediato: al inicio de la tercera ola, el número de mujeres en cargos electivos era escaso. Recién entonces, 
SANTIAGO ALLES

la introducción de cuotas de género, primero en Argentina y luego en una docena de países latinoamericanos (Schwindt-Bayer 2010; Campo 2005), intentó corregir esta situación ${ }^{4}$. Aun cuando este proceso ha dado lugar a una mayor presencia de mujeres en cargos legislativos, el número de legisladoras varía profundamente no sólo a lo largo del tiempo, sino también entre casos: si bien la proporción de mujeres ha crecido en las cinco democracias incluidas en el análisis, la presencia de mujeres en las legislaturas nacionales hoy oscila entre el 8,8\% (Brasil-D) y el 37,5\% (Argentina-S).

TABLA III

ELECCIÓN DE MUJERES LEGISLADORAS, EN CINCO DEMOCRACIAS: ARGENTINA, BRASIL, CHILE, PerÚ y URUguay, 1980-2013. REgRESIÓN BINOMIAL

\begin{tabular}{|c|c|c|c|c|c|c|}
\hline \multirow{2}{*}{ VARIABLES } & \multicolumn{3}{|c|}{ MODELO \#1 } & \multicolumn{3}{|c|}{ MODELO \#2 } \\
\hline & $\beta$ & $\sigma_{\beta}$ & VALOR-Z & $\beta$ & $\sigma_{\beta}$ & VALOR-Z \\
\hline \multicolumn{7}{|c|}{ Variables Independientes } \\
\hline Ideología partidaria & $-0,208 * * *$ & 0,056 & $-3,707$ & $-0,197 * * *$ & 0,058 & $-3,408$ \\
\hline Lista abierta & 0,363 & 0,444 & 0,817 & 0,647 & 0,561 & 1,152 \\
\hline Magnitud partidaria & 0,008 & 0,012 & 0,684 & 0,008 & 0,013 & 0,594 \\
\hline NEP electoral & $0,156^{* * * *}$ & 0,045 & 3,508 & $0,137^{* * *}$ & 0,046 & 2,986 \\
\hline NEP legislativo & $-0,231 * * *$ & 0,058 & $-3,945$ & $-0,205 * *$ & 0,062 & $-3,289$ \\
\hline Magnitud de distrito & 0,001 & 0,004 & 0,199 & 0,000 & 0,004 & $-0,095$ \\
\hline \multicolumn{7}{|c|}{ Términos Interactivos } \\
\hline Cuotas x Lista abierta & $-1,483 * * *$ & 0,210 & $-7,055$ & $-1,779 * * *$ & 0,257 & $-6,923$ \\
\hline Cuotas x Ideología & $-0,018$ & 0,073 & $-0,252$ & $-0,009$ & 0,074 & $-0,115$ \\
\hline Cuotas x Mag. Partidaria & 0,005 & 0,013 & 0,367 & 0,009 & 0,013 & 0,684 \\
\hline Abierta x Mag. partidaria & $-0,039 * *$ & 0,014 & $-2,813$ & $-0,046 * *$ & 0,015 & $-3,123$ \\
\hline Abierta x Ideología & 0,078 & 0,072 & 1,094 & 0,057 & 0,074 & 0,766 \\
\hline \multicolumn{7}{|c|}{ Variables de Control } \\
\hline Cuotas de género & $2,101 * * *$ & 0,467 & 4,501 & $2,182^{* * *}$ & 0,501 & 4,355 \\
\hline Cámara legislativa & 0,010 & 0,123 & 0,085 & 0,003 & 0,128 & 0,027 \\
\hline Analfabetismo (\%) & $-0,058 * * *$ & 0,012 & $-4,819$ & $-0,063 * * *$ & 0,013 & $-4,701$ \\
\hline Nivel de democracia (FH) & $-0,278 * * *$ & 0,070 & $-3,967$ & $-0,310 * * * *$ & 0,077 & $-4,014$ \\
\hline Efectos fijos & No & \multicolumn{5}{|c|}{ Sí } \\
\hline Constante & $-0,833^{*}$ & 0,383 & $-2,175$ & $-0,728$ & 0,397 & $-1,836$ \\
\hline AIC & $2.878,7$ & & & $2.881,5$ & & \\
\hline \multirow[t]{2}{*}{ Observaciones } & 2.894 & & & 2.894 & & \\
\hline & & & & \multicolumn{3}{|c|}{$* * * \mathrm{p}<, 001 * * \mathrm{p}<, 01 * \mathrm{p}<, 05$} \\
\hline
\end{tabular}

Fuente: Elaboración propia.

4. Al mismo tiempo, un pequeño (pero no despreciable) número de partidos en la región han introducido cuotas partidarias (QUOTA PROJECT 2013). 

EN CINCO DEMOCRACIAS LATINOAMERICANAS. .

¿Qué factores explican tal variación entre países con trayectorias políticas y niveles de desarrollo similares? La evidencia señala, tal como anticipa toda la literatura sobre elección de mujeres en cargos legislativos, que las cuotas de género son la explicación principal (véase Tabla III), pero más allá del efecto de las acciones afirmativas, tanto la posición ideológica de las bases partidarias como el tipo de lista tienen efectos significativos sobre las oportunidades electorales de las mujeres; mientras que, por el contrario, la magnitud partidaria no parece tener ningún efecto una vez que se controlan las demás posibles causas. El número de partidos presenta, por su parte, una relación más compleja: el mayor número de competidores en la arena electoral tiene un efecto contrario al número de partidos que obtienen bancas en el distrito.

Más significativo aún, los resultados de la segunda ecuación, que incluye efectos fijos, no difieren de la ecuación más simple, lo cual sugiere que los resultados son robustos: los coeficientes no son producto de elementos idiosincráticos no observados. No obstante, la naturaleza no lineal del modelo estadístico, junto con el número de términos interactivos introducidos en las ecuaciones, hacen que la lectura de resultados no sea intuitiva. Por esa razón, en las siguientes figuras se representan los efectos sustantivos de las variables de interés, manteniendo las variables omitidas en las medias observadas.

\section{FIGURA I}

PROBABILIDAD ESPERADA DE LA ELECCIÓN DE UNA MUJER E INTERVALOS DE CONFIANZA, SEGÚN IDEOLOGÍA PARTIDARIA

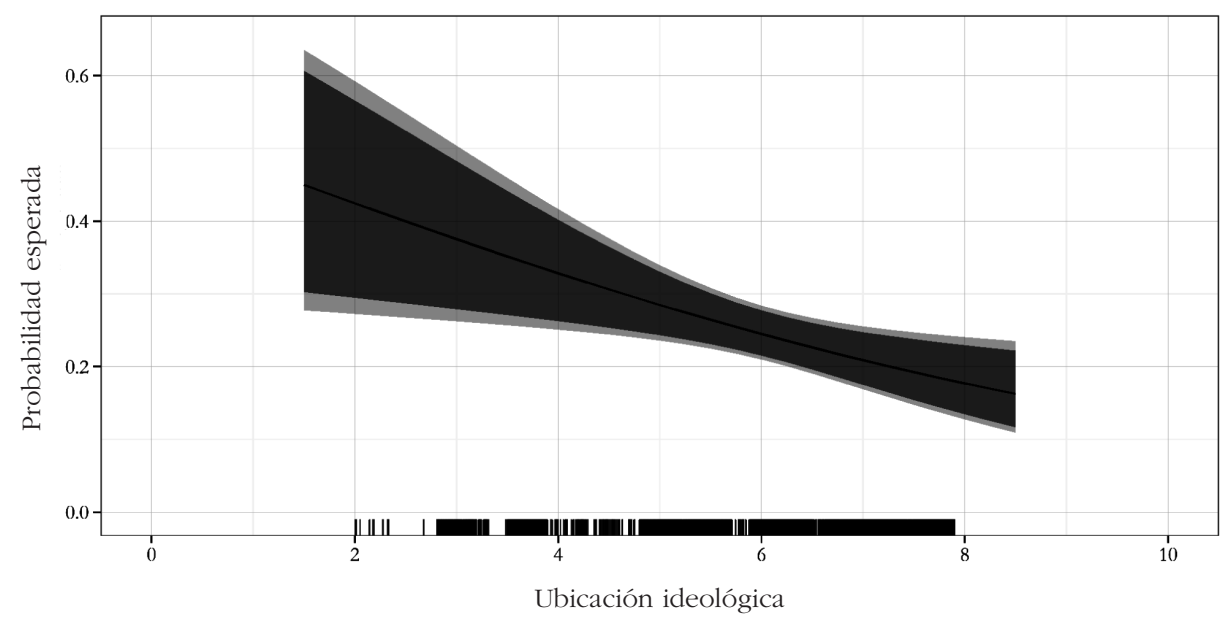

Nota: Las variables omitidas fueron fijadas en sus valores promedio, para una elección con cuotas. Las probabilidades han sido estimadas a partir del Modelo 2; intervalos de confianza al $90 \%$ y $95 \%$. El rug representa la distribución (jiterizada) de la posición ideológica de las observaciones.

Fuente: Elaboración propia. 

EN CINCO DEMOCRACIAS LATINOAMERICANAS...

La posición ideológica de las bases partidarias, tal como puede observarse en la Figura I, tiene un efecto significativo sobre las posibilidades electorales de las mujeres. Por un lado, en una elección sin cuotas, utilizando listas cerradas, manteniendo constantes en sus valores promedio el resto de las variables incluidas en el modelo, la probabilidad esperada de elegir una mujer para un partido de izquierda (ubicado en 2,5 , en una escala $0-10$ ) es del 7,0\%; mientras que la misma probabilidad para un partido ubicado en el centro es del 4,4\%, y para un partido de derecha (7,5 en la misma escala) cae a sólo un 2,7\%.

No obstante, esas diferencias no son estadísticamente significativas. Por otro lado, la probabilidad esperada de elegir una mujer para el mismo partido de izquierda es seis veces mayor para una elección bajo un régimen de cuotas: 40,0\%; mientras que tal probabilidad para el mismo partido de derecha cae a 19,2\%. Es decir, cada punto de desplazamiento a la izquierda incrementa aproximadamente el 4,2\% las chances de elegir una mujer. Estas probabilidades indican que, en una elección legislativa bajo un régimen de cuotas, por cada cinco escaños ganados, mientras un partido de izquierda elegirá dos mujeres, un partido de derecha sólo elegirá una.

En la Figura II se simulan las probabilidades predichas por partido político a partir de su posición ideológica concreta, a fin de brindar predicciones para posiciones programáticas realmente observadas (Hanmer y Kalkan 2013) y no sólo para valores promedio, en una situación hipotética donde todos compitieran bajo las mismas reglas, en contextos sociales idénticos, a partir del primer modelo. Es decir, la figura no presenta promedios, sino predicciones obtenidas de la segunda ecuación (Tabla III), a partir de la posición ideológica de sus bases electorales.

La evidencia sugiere que, en los sistemas partidarios donde existen partidos de izquierda bien definidos, las mujeres encuentran en ellos una avenida principal para el acceso a posiciones electivas. Así, mientras los partidos políticos más importantes en Argentina y en Perú no parecen ofrecer diferencias sustantivas en su apertura a la inclusión de mujeres; el PT brasileño o el Frente Amplio (FA) uruguayo son más favorables que otros partidos en esas mismas arenas. Entre los partidos chilenos, por su parte, dada su distribución a lo largo del continuo ideológico, las oportunidades electorales de las mujeres cambian también gradualmente entre partidos; no obstante lo cual las diferencias entre partidos a la izquierda (PS, PPD) y derecha (UDI) son estadísticamente significativas. En suma, si el PT o el FA no son canales de inclusión más dinámicos, ello se debe en parte a que compiten bajo reglas electorales que deprimen la elección de mujeres; mientras que si el peronismo argentino luce como un partido favorable a las mujeres en comparación a otros partidos latinoamericanos, ello está asociado al uso de cuotas de género con listas cerradas, antes que a distintivas posiciones ideológicas de sus bases electorales. La ideología de las bases partidarias juega un papel sustantivo en la elección de mujeres legisladoras. 


\section{FIGURA II \\ PROBABILIDAD ESPERADA DE LA ELECCIÓN DE UNA MUJER E INTERVALOS DE CONFIANZA, SEGÚN IDEOLOGÍA PARTIDARIA, PARA PARTIDOS POLÍTICOS PRINCIPALES}

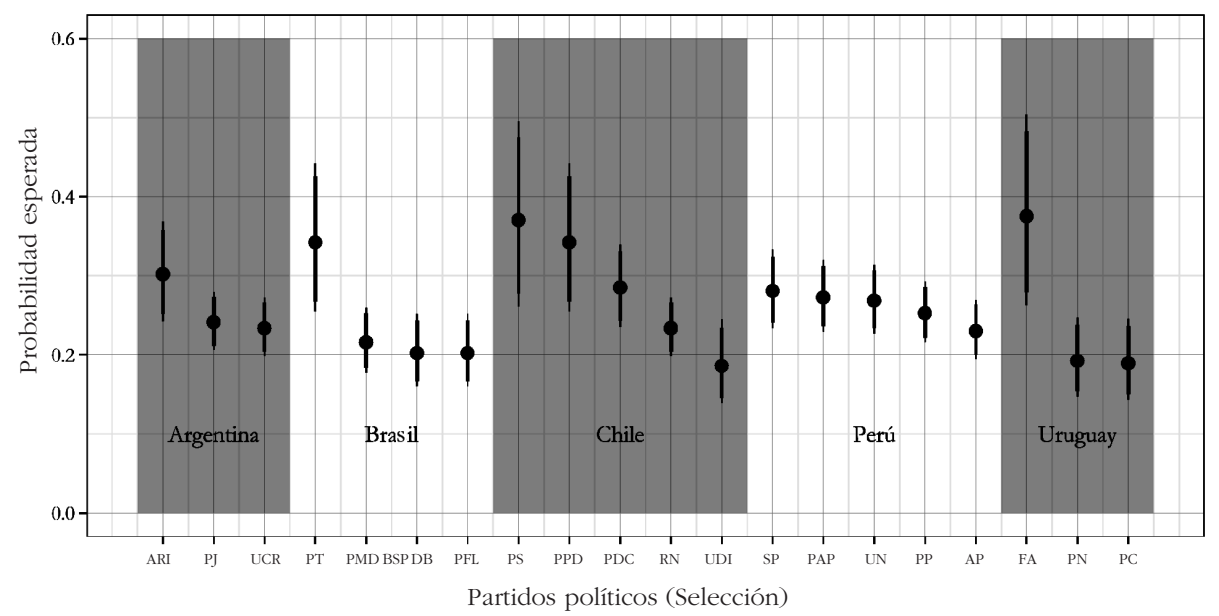

Nota: Las siglas partidarias corresponden a los siguientes partidos: Argentina. ARI: Alianza Alternativa por una República de Iguales; PJ: Partido Justicialista; UCR: Unión Cívica Radical. Brasil. PT: Partido dos Trabalhadores; PMBD: Partido do Movimiento Democrático Brasileiro; PSDB: Partido da Social Democracia Brasileira; PFL: Partido da Frente Liberal. Chile. PS: Partido Socialista; PPD: Partido por la Democracia; PDC: Partido Demócrata Cristiano; RN: Renovación Nacional; UDI: Unión Demócrata Independiente. Perú. SP: Somos Perú; PAP: Partido Aprista Peruano; Un: Unidad Nacional; PP: Perú Posible; AP: Acción Popular. Uruguay. FA: Frente Amplio; PN: Partido Nacional; PC: Partido Colorado.

Las variables omitidas fueron fijadas en sus valores promedio, para una elección con cuotas. Las probabilidades han sido estimadas a partir del Modelo 2; intervalos de confianza al 90\% y $95 \%$.

Fuente: Elaboración propia.

¿Cuál es el efecto de la magnitud sobre las chances de las mujeres? Una vez que se controla el efecto del tamaño de la delegación partidaria, la magnitud de distrito no presenta ninguna influencia significativa: el coeficiente es estadísticamente indistinguible de cero en ambas ecuaciones. Esto no presenta, sin embargo, ningún conflicto con la literatura antecedente: el tamaño de los distritos influye en la elección de legisladoras si hace que las delegaciones partidarias sean más grandes. Más significativo es, no obstante, que el coeficiente de la magnitud partidaria sea también indistinguible de cero, porque sugiere que la cantidad de bancas obtenidas por un partido en un distrito no tiene efecto sobre las probabilidades de elección. A fin de confirmar estos resultados, se estimó el efecto para cada posible magnitud partidaria (hasta 10 escaños, donde las magnitudes mayores fueron agrupadas), cuyos resultados se presentan en la Figura III. 


\section{FIGURA III}

PROBABILIDAD ESPERADA DE LA ELECCIÓN DE UNA MUJER, E INTERVALOS DE CONFIANZA, SEGÚN MAGNITUD DE LA DELEGACIÓN PARTIDARIA Y USO DE CUOTAS DE GÉNERO

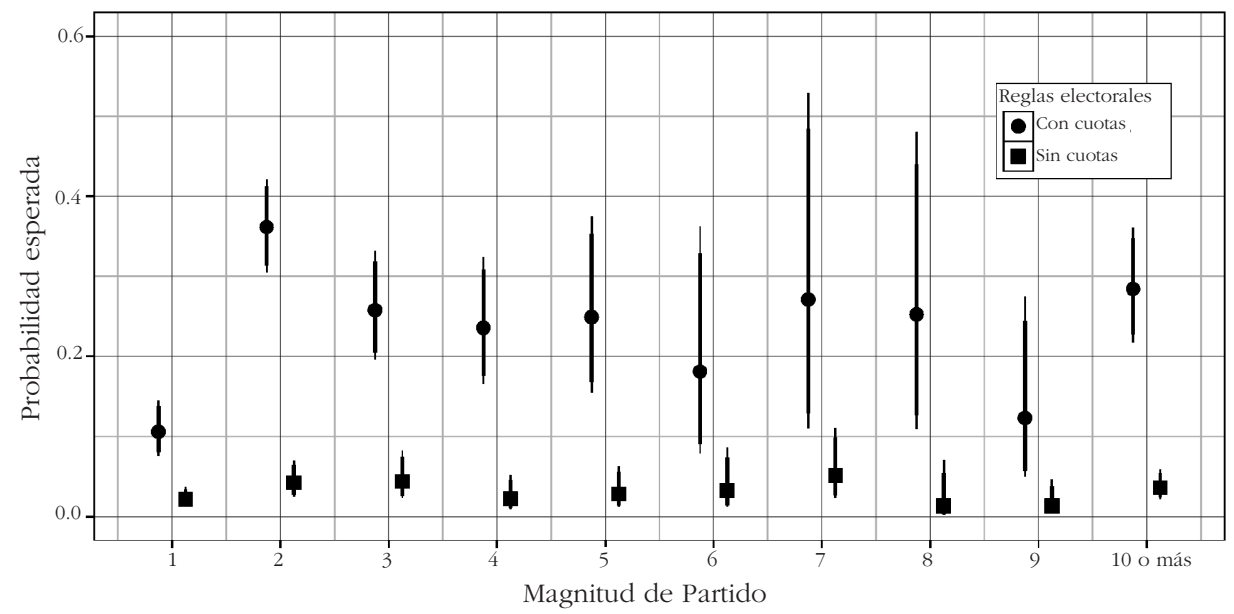

Nota: Las variables omitidas fueron fijadas en sus valores promedio. La estimación corresponde a un modelo no reportado, donde se introdujo una variable dummy para cada magnitud predicha. Se representan intervalos de confianza al $90 \%$ y $95 \%$.

Fuente: Elaboración propia.

Tal como puede observarse, el cambio más significativo ocurre cuando, en una elección con cuotas de género y listas cerradas, la magnitud pasa de una a dos bancas: la probabilidad trepa entonces del $10,6 \%$ al $36,2 \%$. Tras ese incremento, las probabilidades esperadas no muestran ningún patrón consistente, sino que por el contrario oscilan en torno al 25\%; al tiempo que los intervalos de confianza son cada vez más grandes, lo cual indica que las predicciones se vuelven cada vez más inciertas. El patrón es similar entre las elecciones sin cuotas, aunque en este caso no se observan diferencias sustantivas, al eludir delegaciones unipersonales las probabilidades predichas son estadísticamente indistinguibles entre cualquier categoría. El coeficiente observado en ambas ecuaciones se explica, entonces, por ese patrón errático observado en la figura anterior.

Sin embargo, en este punto cabe una aclaración sobre cómo interpretar esos resultados. La Figura III representa la probabilidad de que un legislador electo sea mujer, lo que implica que esa probabilidad se repite tantas veces como escaños compongan la delegación partidaria: por ejemplo, mientras la probabilidad de elegir una mujer es del $77,3 \%$ en una delegación de tres miembros, esa probabilidad predicha trepa al $94,2 \%$ en delegación de cuatro; y espera entre tres y cuatro mujeres electas cuando un partido logra 12 escaños. Es decir, los resultados señalan que cuanto mayor es el número de 
EN CINCO DEMOCRACIAS LATINOAMERICANAS...

bancas obtenido, mayor será el número de mujeres electas; pero más importante aún, por un lado, indican que, ese número no crece en forma lineal ni monotónica y, por otro, sugiere, que una vez que se introducen cuotas de género, el primer obstáculo a vencer son las delegaciones unipersonales. Los resultados muestran cómo los líderes partidarios, tal como dan cuenta Matland y Taylor (1997) en el caso costarricense, reservan el primer lugar para un hombre; y ese juego de suma cero sólo se relaja una vez que el número de escaños es mayor a uno.

FIGURA IV

PROBABILIDAD ESPERADA DE LA ELECCIÓN DE UNA MUJER E INTERVALOS DE CONFIANZA, SEGÚN FRAGMENTACIÓN ELECTORAL

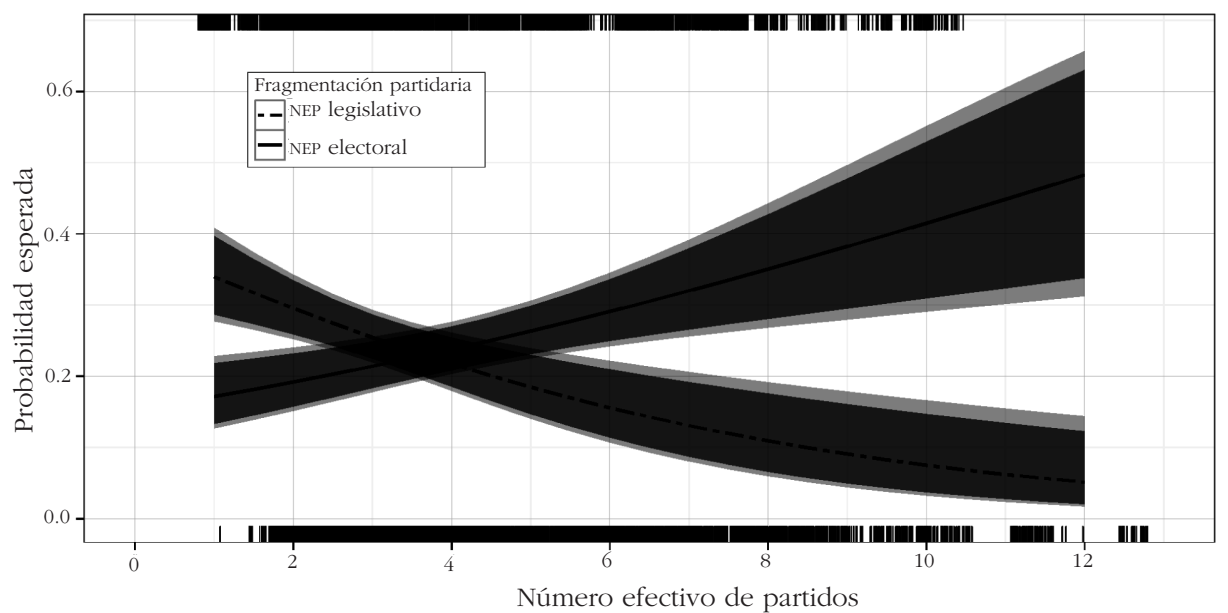

Nota: Las variables omitidas fueron fijadas en sus valores promedio, para una elección con cuotas. Las probabilidades han sido estimadas a partir del Modelo 2; intervalos de confianza al 90\% y 95\%. Los rugs representan la distribución (jiterizada) del número efectivo de partidos electoral (votos: abajo) y legislativo (escaños: arriba) en las observaciones.

Fuente: Elaboración propia.

Por otra parte, los resultados reportados en ambas ecuaciones en la Tabla III indican que la fragmentación de la competencia electoral tiene efecto sobre la elección de legisladoras, aun cuando se controla el efecto de la magnitud partidaria: mientras el mayor número de competidores en la arena electoral mejora las chances de elegir una mujer, el número de partidos que obtienen escaños está asociado negativamente con la elección de mujeres. Sin embargo, estos dos tipos de fragmentación varían usualmente en sintonía, por lo cual ambos efectos con frecuencia se cancelan mutuamente. No obstante, cuando los efectos reductores de las reglas electorales son fuertes, la fragmentación electoral guarda una relación débil con la fragmentación legislativa. 
El mejor escenario para la elección de mujeres, entonces, parece ser uno donde los efectos reductores del sistema electoral son muy pronunciados. En esta situación, los líderes partidarios esperan una competencia electoral intensa y la nominación de una mujer puede ser una forma de diferenciación, por lo cual, tal como afirman Matland y Studlar (1996), el «contagio» entre partidos es más probable.

En suma, la magnitud de distrito puede eventualmente tener un efecto indirecto sobre las probabilidades de elección, pero los mecanismos causales que conectan magnitud y elección son complejos. Por un lado, cuando un partido pasa de obtener un escaño a obtener dos, las chances de elegir una mujer cambian sustantivamente, siempre que las elecciones se lleven a cabo bajo un régimen de cuotas y con listas cerradas (no así cuando alguna de estas condiciones está ausente). Más allá de este punto, las probabilidades son relativamente constantes, por lo cual el número de mujeres electas será un resultado mecánico del total de escaños obtenidos. Por otro lado, la mayor cantidad de actores partidarios no parece ejercer por sí mismo presión sobre la elección de mujeres en cargos legislativos, sino que por el contrario es la mayor intensidad de la competencia electoral, impuesta por reglas con fuertes efectos reductores, la que impulsa la elección de mujeres.

\section{FIGURA V}

PROBABILIDAD ESPERADA DE LA ELECCIÓN DE UNA MUJER, E INTERVALOS DE CONFIANZA, SEGÚN USO DE CUOTAS Y TIPO DE LISTA

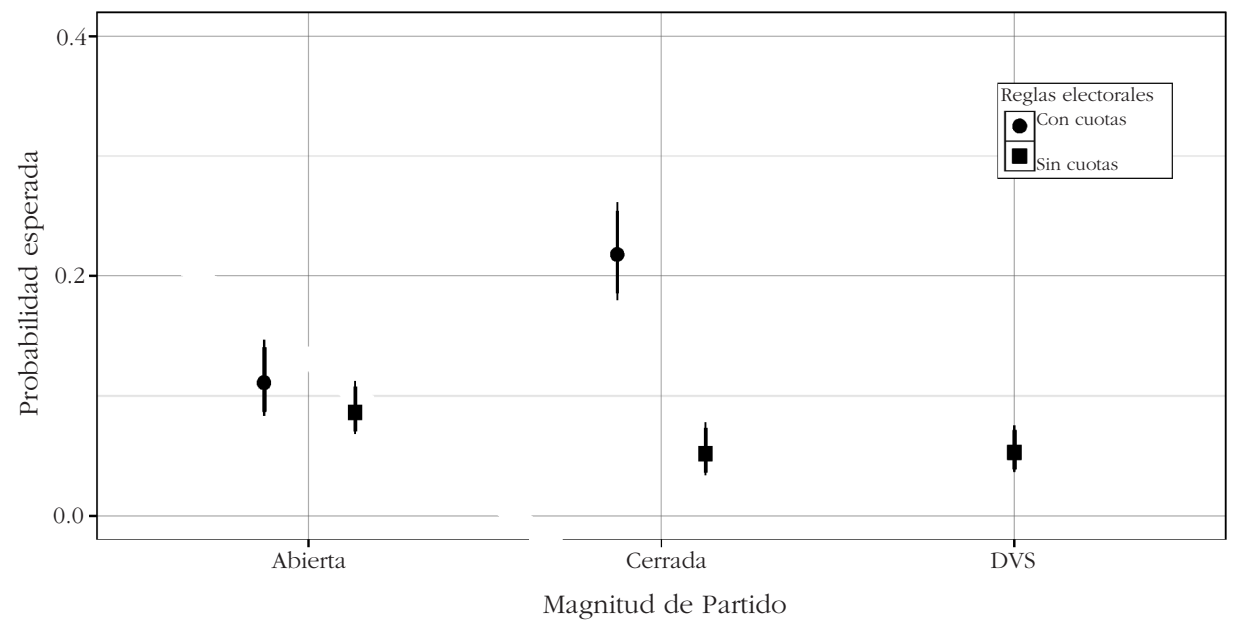

Nota: Las variables omitidas fueron fijadas en sus valores promedio. La estimación corresponde a un modelo no reportado; intervalos de confianza al $90 \%$ y $95 \%$.

Fuente: Elaboración propia. 
EN CINCO DEMOCRACIAS LATINOAMERICANAS. .

El tipo de lista de candidatos tiene efectos sustantivos sobre las oportunidades electorales de las mujeres, aunque sus efectos, tal como han argumentado trabajos anteriores (Jones 2009; Jones et al. 2012), son condicionales al uso de cuotas (véase Figura IV). En este caso, en el modelo no se han incluido efectos fijos por país, a fin de evitar problemas de multicolinealidad, pero se han introducidos efectos fijos por década: los resultados son consistentes a través de las especificaciones. Por un lado, el tipo de lista tiene efectos escasos sobre las probabilidades de elección cuando no existen cuotas de género, aunque en este contexto las listas abiertas son levemente más favorables que las listas cerradas: mientras la probabilidad de elegir una mujer es del 5,2\% para una lista cerrada; la probabilidad alcanza un $8,6 \%$ en una elección con lista abierta 5 .

Por el contrario, las listas cerradas son significativamente más favorables cuando se combinan con leyes de cuotas, lo que implica que la implementación de cuotas de género se ve significativamente afectada por el uso de listas abiertas: mientras la probabilidad de elegir una mujer es del $21,8 \%$ con listas cerradas, esa probabilidad cae al $11,1 \%$ cuando la elección se realiza con listas abiertas. Más aún, las elecciones con listas abiertas prácticamente eliminan los cambios introducidos por las cuotas de género: el efecto es estadísticamente indistinguible.

Por último, entre las variables de control incluidas en los modelos, una merece algunos comentarios adicionales: los niveles de democracia están asociados con las oportunidades electorales de las mujeres. A medida que aumentan el valor del índice integrado de Freedom House (es decir, a medida que se deteriora el nivel de democracia), las probabilidades de elegir una mujer como legisladora declinan en forma significativa. Así, mientras la probabilidad esperada de acceder a una banca para una mujer es del $32,3 \%$ en una democracia plena $(\mathrm{FH}=1,0)$ bajo un régimen de cuotas, tal probabilidad se reduce al 23,0\% cuando el score declina al mínimo considerado «libre» $(\mathrm{FH}=2,5)$; y a sólo el $12,1 \%$ cuando el score llega al piso de los regímenes considerados parcialmente libres $(\mathrm{FH}=5,0)$. Las diferencias entre esas tres situaciones son estadísticamente significativas (con una confianza del 95\%). En suma, las oportunidades de las mujeres parecen mejorar sustantivamente a medida que se consolida la democracia.

\section{DISCUSIÓN}

El foco central del presente trabajo ha sido analizar el efecto de la ideología partidaria sobre las probabilidaes electorales de las mujeres. La literatura antecedente (Kenworthy y Malami 1999; Reynolds 1999; Matland 1993; Matland y Studlar 1996; Jones 2009; Schmidt 2009; Sanbonmatsu 2002) ha dado cuenta, a partir de datos agregados, cómo el número de mujeres electas crece con los votos y con los escaños de

5. A su vez, las listas por facción tienen un comportamiento muy similar a las listas cerradas, con una probabilidad de elegir una mujer del 5,3\%, estadísticamente indistinguible.

6. Los resultados son consistentes cuando se utiliza el índice Polity IV como un indicador alternativo para medir el nivel de democracia. 
partidos de izquierda. Los hallazgos aquí presentados, en esa misma dirección, dan una medida precisa del impacto de la ideología partidaria: en una escala izquierda-derecha de 0 a 10, por cada punto a la izquierda, las probabilidades de elegir una mujer crecen un $4,2 \%$, lo que significa que las chances entre un partido de izquierda y uno de derecha difieren en alrededor de un 20,8\%. Tal efecto, sin embargo, sólo es estadísticamente significativo en las elecciones con cuotas de género; mientras que la evidencia (aun cuando el efecto presenta el mismo sentido) no permite hacer aseveraciones concluyentes en las elecciones sin cuotas.

La evidencia presentada, al tiempo que contribuye a la literatura dando una medida precisa del efecto de la ideología partidaria, abre la puerta a nuevos interrogantes. Los procesos de nominación siguen siendo, para usar la metáfora de Gallagher y Marsh (1988), un «jardín secreto de la política» y a pesar de los esfuerzos más recientes en la investigación comparada (Hazan y Rahat 2010; Rahat y Hazan 2001; Freidenberg y Alcántara Sáez 2009), la literatura (salvo contadas excepciones, Caul 1999) ha dedicado escasa atención al papel de la organización partidaria en la elección de mujeres. El estudio de los procesos de nominación puede echar luz sobre los mecanismos causales que conectan ideología partidaria y elección: la evidencia aquí presentada permite afirmar que los partidos de izquierda son más favorables a la elección de mujeres, pero no permite distinguir si ello es consecuencia de una mayor incorporación de las mujeres en sus élites, de una mayor apertura de sus élites con independencia de la presencia de mujeres o de una mayor predisposición de los electores a votar mujeres.

El siguiente desafío consiste en modelar la distribución ideológica de los votantes y de las élites partidarias por separado, de manera que permita establecer cómo las élites interactúan con los electores. Estos mecanismos causales son diferenciables en términos teóricos: al momento de nominar candidatos, ¿los partidos progresistas se vuelven «más conservadores» cuando compiten en distritos conservadores?, ¿y los partidos conservadores se vuelven «más progresistas» en distritos más liberales? Estos mecanismos resultan hasta aquí difíciles de distinguir, dados tanto potenciales problemas de endogeneidad, como así también dadas limitaciones en términos de evidencia, pero tales obstáculos no son, sin embargo, insalvables.

A pesar de la insistencia en la literatura (Matland 1993; Matland y Taylor 1997; Norris 2004; Jones 1998; Rule 1987; Alles 2008; también Paxton et al. 2007) sobre las ventajas de los distritos grandes, los hallazgos aquí reportados indican que su efecto es menor. El mecanismo causal propuesto indica que la magnitud de distrito está positivamente asociada con las magnitudes partidarias y con la fragmentación electoral, no obstante estos mecanismos no operan tal como sugiere el argumento. El efecto más importante de la magnitud partidaria es condicional a un específico set de reglas (cuotas+listas cerradas) y está considerablemente limitado a las magnitudes mínimas: manteniendo las variables omitidas en sus medias observadas, si un partido obtiene dos bancas en dos distritos diferentes, la chance de que una de ellas sea para una mujer ronda el 25\%; pero si esas bancas componen la misma delegación partidaria, las chances de que haya una mujer entre los dos electos escala al $78 \%$.

Tras ese umbral, no obstante, el número de mujeres electas crece con probabilidades relativamente estables y estadísticamente indiferenciables, como resultado 

EN CINCO DEMOCRACIAS LATINOAMERICANAS. .

mecánico del tamaño de la delegación. La estructura de la competencia partidaria sólo juega un papel sustantivo cuando en un distrito conviven una alta fragmentación electoral y una baja fragmentación legislativa, gracias a un sistema electoral con sólidos efectos reductores. En suma, analizando un extenso set de datos, los hallazgos del presente trabajo moderan, en línea con trabajos recientes (Jones 2009; Schmidt 2009; Jones et al. 2012), las aseveraciones sobre los efectos de la magnitud de distritos, pero al mismo tiempo dan considerable crédito a la idea de «contagio» ( $c f r$. Matland y Studlar 1996): la presión para la inclusión de mujeres, aun cuando se controla la cantidad de bancas logradas, es mayor donde el número de partidos electorales es alto pero pocos partidos logran representación parlamentaria, es decir, en los distritos donde la competencia electoral es más intensa y mayor la demanda, se produce la diferenciación en la oferta.

Las listas abiertas, contrariamente a lo afirmado por trabajos anteriores (Schmidt 2009; Matland 2006 y 2005; Schmidt y Saunders 2004; Shugart 1994), perjudican las oportunidades electorales de las mujeres. No obstante, la superioridad de las listas cerradas, tal como trabajos anteriores han señalado (Jones 2009; Jones et al. 2012), es condicional a su interacción con cuotas de género. Estos hallazgos sugieren que la competencia intrapartidaria es una barrera más alta que el tamaño de la delegación partidaria, lo cual es consistente con la literatura precedente (Schwindt-Bayer 2009; Jones 2009; Jones et al. 2012).

Sin embargo, cuando las élites partidarias no se ven obligadas a incluir mujeres en posiciones expectables en las listas de candidatos, las listas abiertas al menos permiten a las mujeres buscar el apoyo de los votantes; mientras que las listas cerradas dejan su suerte atada a las decisiones de las autoridades partidarias. Más aún, la interacción con las cuotas de género indica que la efectividad de las acciones afirmativas, tal como sugiere la literatura anterior (Jones 2004; Dahlerup 2005; Htun y Jones 2002), está condicionada por otros elementos del diseño de las instituciones electorales.

Hasta aquí se ha presentado evidencia preliminar de los efectos del nivel de democracia sobre las oportunidades electorales de las mujeres, lo cual habría sido difícil de observar. La intuición teórica sugiere que la consolidación de la democracia va acompañada por la consolidación de visiones más igualitarias de la participación ciudadana, se trate de géneros, clases sociales o grupos étnicos. Tal consolidación de la igualdad, precisamente, debe reducir las aprehensiones de los votantes a elegir a mujeres en cargos legislativos y favorecer la igualdad de género en las instituciones representativas.

No obstante, con regularidad, los trabajos cuantitativos (Kenworthy y Malami 1999; Reynolds 1999; Tripp y Kang 2008; Paxton 1997; Paxton y Kunovich 2003; Kunovich y Paxton 2005) no han encontrado ningún efecto del nivel de democracia sobre la elección de mujeres o incluso, en algunos casos, han encontrado efectos negativos; mientras la literatura cualitativa (Waylen 1994; Jaquette y Wolchik 1998) ha reportado un declive en la participación política de las mujeres en el periodo postransición. Sólo recientemente Fallon y coautores (2012) han encontrado evidencia: los efectos habrían sido escurridizos como resultado de las diferencias entre niveles de desarrollo, lo cual sugiere que no sólo los efectos de las reglas electorales (Matland 1998) sino también los del propio régimen político son condicionales al nivel de desarrollo. 
Los resultados reportados, por un lado, están en línea con los obtenidos por Fallon y coautores (2012): las chances de resultar electa para una mujer son sustantivamente mejores en una democracia consolidada que en otra en consolidación; y cuanto mayor es el deterioro democrático, menor será la paridad entre hombres y mujeres. Por otro lado, estos hallazgos además sugieren que los diseños transversales en un solo momento, tal como han indicado sus críticos (Hughes y Paxton 2007; Roberts et al. 2013), pueden resultar problemáticos, porque corren el riesgo de perder de vista que los tiempos de la incorporación difieren de un país a otro. No obstante, estos resultados son preliminares y deben ser tomados con cautela, tanto por lo reducido del set de casos, como por la limitada variación en el nivel de democracia entre los casos analizados: una exploración más completa requiere mayor variación entre casos y entre elecciones.

Las últimas décadas han sido testigo de una creciente participación de mujeres en cargos electivos, tanto nacionales como subnacionales, en todos los países de la región: mientras en los años inmediatamente posteriores a las transiciones democráticas unas pocas «gigantes» (Matland 1993) ocupaban cargos electivos, en la actualidad una de cada cinco bancas legislativas está en poder de una mujer (IPU 2013). La transformación, aun cuando sigue siendo insuficiente, ha sido enorme. No obstante, una observación más detenida de esos mismos casos muestra diferentes niveles de incorporación, como así también diferentes ritmos en ese proceso. ¿Qué explica esas diferencias? El presente trabajo ha brindado evidencia del efecto de factores partidarios (ideología) e institucionales (tipo de listas), al tiempo que ha aportado datos y análisis que moderan las afirmaciones realizadas por una parte de la literatura sobre otros factores, tales como la magnitud de distrito y la magnitud partidaria, a la vez que ha reportado la compleja relación que presenta la fragmentación partidaria.

\section{BIBLIOGRAFÍA}

Alcántara SÁez, Manuel y Freidenberg, Flavia (eds.). Partidos políticos de América Latina. Salamanca: Ediciones Universidad de Salamanca, 2001.

ALLES, Santiago. ¿Hacia la consolidación política? Cambios en la «Estructura de oportunidades electorales» de las mujeres en Argentina. América Latina Hoy, Revista de Ciencias Sociales, 2007, vol. 47: 123-154.

ALLES, Santiago. Efectos del Sistema Electoral sobre la Representación de Mujeres. Argumentos y Evidencia a partir del caso argentino (1983-2005). Revista SAAP, 2008, vol. 3 (2): 313-352.

AMES, Barry. Electoral strategy under open-list proportional representation. American Journal of Political Science, 1995, vol. 39 (2): 406-433.

ARCHENTI, Nélida y TulA, María Inés. Algunas cuestiones iniciales sobre las leyes de cuotas. En ARCHEnti, Nélida y Tula, María Inés. Mujeres y Política en América Latina. Sistemas electorales y cuotas de género. Buenos Aires: Heliasta, 2008: 9-29.

BHAVNANI, Rikhil R. Do Electoral Quotas Work after They Are Withdrawn? Evidence from a Natural Experiment in India. American Political Science Review, 2009, vol. 103 (1): 23-35.

BUQUET, Daniel. El doble voto simultáneo. Revista SAAP, 2003, vol. 1 (2): 317-339.

CAMPO, Esther del. Women and Politics in Latin America: Perspectives and Limits of the Institutional Aspects of Women's Political Representation. Social Forces, 2005, vol. 83 (4): 1697-1725. 
CAREY, John y SHUGART, Matthew S. Incentives to cultivate a personal vote: a rank ordering of electoral formulas. Electoral Studies, 1995, vol. 14 (4): 417-439.

CAUL, Miki. Women's representation in parliament: the role of political parties. Party Politics, 1999, vol. 5 (1): 79-98.

CHILDS, Sarah y KROOK, Mona Lena. Analyzing Women's Substantive Representation: From Critical Mass to Critical Actors. Government \& Opposition, 2009, vol. 44 (2): 125-145.

COLOMER, Josep M. y ESCATEL, Luis E. La dimensión izquierda-derecha en América Latina. Desarrollo Económico, 2005, vol. 45 (177): 123-136.

Cox, Gary. Making Votes Count: Strategic Coordination in the World's Electoral Systems. Cambridge University Press, 1997.

DAHLERUP, Drude. From a Small to a Large Minority: Women in Scandinavian Politics. Scandinavian Political Studies, 1988, vol. 11 (4): 275-298.

- Estudios comparativos sobre las cuotas de género. La aplicación de las cuotas: experiencias latinoamericanas. Resumen del Taller. International IDEA, 2003.

- Increasing Women's Political Representation: New Trends in Gender Quotas. En BALLINGTON, Julie y KARAM, Azza. Women in Parliament: Beyond Numbers. International Institute for Democracy and Electoral Assistance. Stockholm: IDEA, 2005: 141-153.

DAHLERUP, Drude y FREIDENVALL, Lenita. Quotas as «Fast Track» to Equal Representation for Women. International Feminist Journal of Politics, 2005, vol. 7 (1): 26-48.

FALLON, Kathleen; SwISS, Liam y VITERNA, Jocelyn. Resolving the Democracy Paradox: Democratization and Women's Legislative Representation in Developing Nations, 1975 to 2009. American Sociological Review, 2012, vol. 77 (3): 380-408.

FRANCESCHET, Susan y PISCOPO, Jennifer M. Gender Quotas and Women's Substantive Representation: Lessons from Argentina. Politics and Gender, 2008, vol. 4 (3): 393-425.

FreIDENBERG, Flavia y AlCÁNTARA SÁEZ, Manuel (eds.). Selección de candidatos, politica partidista y rendimiento democrático. México, DF: Tribunal Federal Electoral del Distrito Federal, 2009.

Gallagher, Michael y MARSH, Michael. Candidate Selection in Comparative Perspective: The Secret Garden of Politics. Sage, 1988.

Gelman, Andrew y HiLl, Jennifer. Data Analysis Using Regression and Multilevel/Hierarchical Models. Cambridge University Press, 2007.

HANMER, Michael y KaLKan, Kerem Ozan. Behind the Curve: Clarifying the Best Approach to Calculating Predicted Probabilities and Marginal Effects from Limited Dependent Variable Models. American Journal of Political Science, 2013, vol. 57 (1): 263-277.

Hazan, Reuven y RaHAT, Gideon. Democracy Within Parties: Candidate Selection Methods and their Political Consequences. Oxford University Press, 2010.

HTUn, Mala. Mujeres y poder político en América Latina. En Méndez MonTALvo, Myriam y BALlington, Julie. Mujeres en el Parlamento. Más allá de los números. Stockhlm: International IDEA, 2002: 19-43.

HTUn, Mala y JONES, Mark P. Engendering the right to participate in decision-making: Electoral quotas and women's leadership in Latin America. En CRASKE, Nikki y MOLYNEUX, Maxine. Gender and the politics of rights and democracy in Latin America. New York: Palgrave, 2002: 32-56.

Hughes, Melanie y Paxton, Pamela. Familiar Theories from a New Perspective: The Implications of a Longitudinal Approach to Women in Politics Research. Politics and Gender, 2007, vol. 3 (3): 370-378.

INGLEHART, Ronald y NORRIS, Pippa. Rising Tide: Gender Equality and Cultural Change. New York: Cambridge University Press, 2003. 
INGLEHART, Ronald; NORRIS, Pippa y WeLZEL, Christian. Gender Equality and Democracy. Comparative Sociology, 2002, vol. 1 (3-4): 321-345.

InTER-Parliamentary Union. 2013. Women in National Parliaments. Disponible en: http:// www.ipu.org/wmn-e/world.htm, consultado: el 11 de octubre del 2013.

JaquetTe, Jane S. y WolchiK, Sharon L. (eds.). Women and Democracy: Latin America and Central and Eastern Europe. Baltimore, MD: Johns Hopkins University Press, 1998.

JONES, Mark P. Legislator Gender and Legislator Policy Priorities in the Argentine Chamber of Deputies and the United States House of Representatives. Policy Studies Journal, 1997, vol. 25: 613-629.

JONES, Mark P. Gender Quotas, Electoral Laws, and the Election of Women: Lessons From the Argentine Provinces. Comparative Political Studies, 1998, vol. 31 (1): 3-21.

JONES, Mark P. Double Simultaneous Vote. En ROSE, Richard. The International Encyclopedia of Elections. Washington, DC: Congressional Quarterly Inc., 2000, pp. 69-70.

JONES, Mark P. Quota legislation and the election of women: learning from the Costa Rican experience. Journal of Politics, 2004, vol. 66 (4): 1203-1223.

JONES, Mark P. Gender Quotas, Electoral Laws, and the Election of Women. Evidence From the Latin American Vanguard. Comparative Political Studies, 2009, vol. 42 (1): 56-81.

JONES, Mark P.; ALLES, Santiago y TCHINTIAN, Carolina. Cuotas de género, leyes electorales y elección de legisladoras en América Latina. Revista de Ciencia Política, 2012, vol. 32 (2): 331-357.

JONES, Mark P. y NAVIA, Patricio. Assessing the Effectiveness of Gender Quotas in Open-list Proportional Representation Electoral Systems. Social Science Quarterly, 1999, vol. 80 (2): 341-355.

KenWorthy, Lane y Malami, Melissa. Gender Inequality in Political Representation: A Worldwide Comparative Analysis. Social Forces, 1999, vol. 78 (1): 235-269.

KING, Gary; KEOHANE, Robert O. y VERBA, Sidney. Designing Social Inquiry: Scientific Inference in Qualitative Research. Princeton University Press, 1994.

KitTILSON, Miki Caul. Representing Women: The Adoption of Family Leave in Comparative Perspective. Journal of Public Economics, 2008, vol. 70 (2): 323-334.

KROOK, Mona Lena. Beyond Supply and Demand: A Feminist-Institutionalist Theory of Candidate Selection. Political Research Quarterly, 2010, 63(4): 707-720.

Kunovich, Sheri y PaXton, Pamela. Pathways to Power: The Role of Political Parties in Women's National Political Representation. American Journal of Sociology, 2005, vol. 111 (2): 505-552.

LAaKSO, Marku y TAagePERA, Rein. Effective Number of Parties: A Measure with Application to West Europe. Comparative Political Studies, 1979, vol. 12 (1): 3-27.

LEVITSKY, Steven y ROBERTS, Kenneth (eds.). The Resurgence of the Latin American Left. Baltimore, MD: Johns Hopkins University Press, 2011.

LIJPHART, Arend. Comparative Politics and the Comparative Method. American Political Science Review, 1971, vol. 65 (3): 682-693.

MARX, Jutta; BORNER, Jutta y CAMINOTTI, Mariana. Las Legisladoras. Cupos de género y política en Argentina y Brasil. Buenos Aires: Siglo XXI, 2007.

MATLAND, Richard. Institutional Variables Affecting Female Representation in National Legislatures: The Case of Norway. Journal of Politics, 1993, vol. 55 (3): 737-755.

MATLAND, Richard. Women's representation in national legislatures: Developed and developing countries. Legislative Studies Quarterly, 1998, vol. 23 (1): 109-125. 
Matland, Richard. Enhancing Women's Political Participation: Legislative Recruitment and Electoral Systems. En KARAM, Azza. Women in Parliament: Beyond Numbers. Stockholm: International IDEA, 2002: 93-111.

Matland, Richard. Enhancing Women's Political Participation: Legislative Recruitment and Electoral Systems. En BALlington, Julie y Karam, Azza (eds.). Women in Parliament: Beyond Numbers. International Institute for Democracy and Electoral Assistance. Stockholm: IDEA, 2005: 93-110.

MATLAND, Richard. Electoral Quotas: Frequency and Effectiveness. En DAHLERUP, Drude (ed.). Women, Quotas, and Politics. New York: Routledge, 2006: 275-292.

Matland, Richard y StUdlaR, Donley. The Contagion of Women Candidates in Single-Member District and Proportional Representation Electoral Systems: Canada and Norway. Journal of Politics, 1996, vol. 58 (3): 707-733.

MATLAND, Richard y STUDLAR, Donley. Gender and Electoral Opportunity Structure in the Canadian Provinces. Political Research Quarterly, 1998, vol. 51 (1): 117-140.

MATLAND, Richard y TAYLOR ROBINSON, Michelle. Electoral system effects on women's representation. Theoretical arguments and evidence from Costa Rica. Comparative Political Studies, 1997, vol. 30 (2): 186-210.

MolyneuX, Maxime. Women's Movement in International Perspective. Latin America and Beyond. New York: Palgrave, 2001.

NORRIS, Pippa. Women in European Legislative Elites. West European Politics, 1985, vol. 8 (4): 90-101.

NORRIS, Pippa. Electoral Engineering. Voting rules and political behavior. Cambridge, MA: Cambridge University Press, 2004.

NORRIS, Pippa y LOVENDUSKI, Joni. Political Recruitment: Gender, Race, and Class in the British Parliament. Cambridge, MA: Cambridge University Press, 1995.

PaXTon, Pamela. Women in National Legislatures: A Cross-National Analysis. Social Science Research, 1997, vol. 26 (4): 442-464.

PaXton, Pamela y Hughes, Melanie M. Women, politics, and power: A global perspective. Thousand Oaks, CA: Pine Forge Press, 2007.

PAXTon, Pamela; Hughes, Melanie M. y GREEN, Jennifer L. The international women's movement and women's political representation, 1893-2003. American Sociological Review, 2006, vol. 71: 898-920.

PaXton, Pamela; Hughes, Melanie y PAInTER, Matthew. Growth in Women's Political Representation: A Longitudinal Exploration of Democracy, Electoral System, and Gender Quotas. European Journal of Political Research, 2010, vol. 49: 25-52.

PAXTON, Pamela y KunOVICH, Sheri. Women's Political Representation: The Importance of Ideology. Social Forces, 2003, vol. 82 (1): 87-113.

Paxton, Pamela; Kunovich, Sheri y Hughes, Melanie M. Gender in Politics. Annual Review of Sociology, 2007, vol. 33: 263-284.

Quota Project. 2013. Global Database of Quotas for Women. Disponible en: http://www. quotaproject.org/, consultado: 12/24/2013.

RAHAT, Gideon y HAZAN, Reuven. Candidate Selection Methods: An Analytical Framework. Party Politics, 2001, vol. 7 (3): 297-322.

REMmeR, Karen. The Rise of Leftist-Populist Governance in Latin America. The Roots of Electoral Change. Comparative Political Studies, 2012, vol. 45 (8): 947-972.

REYNOLDS, Andrew. Women in the Legislatures and Executives of the World: Knocking at the Highest Glass Ceiling. World Politics, 1999, vol. 51 (4): 547-572. 
ROBERTS, Andrew; SEAWright, Jason y CYR, Jennifer. Do Electoral Laws Affect Women's Representation? Comparative Political Studies, 2013, vol. 46 (12): 1555-1581.

Rosenbluth, Frances; SALMOND, Rob y THIES, Michael. Welfare Works: Explaining Female Legislative Representation. Politics E Gender, 2006, vol. 2 (02): 165-192.

RULE, Wilma. Electoral systems, contextual factors and women's opportunity for election to parliament in twenty-three democracies. Western Political Quarterly, 1987, vol. 40: 477-498.

SALMOND, Rob. Proportional representation and female parliamentarians: Explaining overtime changes across the developed world. Legislative Studies Quarterly, 2006, vol. 31 (2): $175-204$.

Sanbonmatsu, Kira. Political Parties and the Recruitment of Women to State Legislatures. Journal of Politics, 2002, 64 (3): 791-809.

SCHMIDT, Gregory. The election of women in list PR systems: testing the conventional wisdom. Electoral Studies, 2009, vol. 28 (2): 190-203.

SCHMIDT, Gregory y SAUNDERS, Kyle. Effective Quotas, Relative Party Magnitude, and the Success of Female Candidates. Peruvian Municipal Elections in Comparative Perspective. Comparative Political Studies, 2004, vol. 37 (6): 704-724.

SCHWINDT-BAyER, Leslie. Still Supermadres? Gender and the Policy Priorities of Latin American Legislators. American Journal of Political Science, 2006, vol. 50 (3): 570-585.

SCHWINDT-BAYER, Leslie. Making Quotas Work: The Effect of Gender Quota Laws On the Election of Women. Legislative Studies Quarterly, 2009, vol. 34 (1): 5-28.

SCHWINDT-BAYER, Leslie. Political Power and Women's Representation in Latin America. New York: Oxford University Press, 2010.

SHUGART, Matthew. Minorities represented and Unrepresented. En RULE, Wilma y ZIMMERMAN, Joseph F. (eds.). Electoral Systems in Comparative Perspective: Their Impact on Women and Minorities. Westport, CT: Westview Press, 1994: 31-44.

TAagePera, Rein y SHugart, Matthew. Seats and Votes. The Effects and Determinants of Electoral Systems. New Haven, CT: Yale University Press, 1989.

TraIn, Kenneth. Discrete Choice Methods with Simulation. Cambridge University Press, 2009.

TREIER, Shawn y JACKMAN, Simon. Democracy as a Latent Variable. American Journal of Political Science, 2008, vol. 52 (1): 201-217.

TRIPP, Aili Mari y KANG, Alice. The global impact of quotas on the fast track to increased female legislative representation. Comparative Political Studies, 2008, vol. 41 (3): 338-361.

VALDINI, Melody Ellis. A deterrent to diversity: The conditional effect of electoral rules on the nomination of women candidates. Electoral Studies, 2012, vol. 31 (4): 740-749.

WAYLEN, Georgina. Women and Democratization: Conceptualizing Gender Relations in Transition Politics. World Politics, 1994, vol. 46:327-355.

WELCH, Susan y STUDLAR, Donley. Multi-member districts and the representation of women: evidence from Britain and the United States. Journal of Politics, 1990, vol. 52 (2): 391-412.

WEYLAND, Kurt; MADRID, Raul y HUNTER, Wendy (eds.). Leftist Governments in Latin America. Successes and Shortcomings. Cambridge, MA: Cambridge University Press, 2010.

WOLBRECHT, Christina y CAMPBELl, David E. Leading by Example: Female Members of Parliament as Political Role Models. American Journal of Political Science, 2007, vol. 51 (4): 921-939.

ZECHMEISTER, Elizabeth y CORRAL, Margarita. Individual and Contextual Constraints on Ideological Labels in Latin America. Comparative Political Studies, 2013, vol. 46 (6): 675-701. 